\title{
Intensive Care Units: The Clinical Pharmacist Role to Improve Clinical Outcomes and Reducing Mortality Rate an Undeniable Function
}

\author{
Luisetto $\mathbf{M}^{* 1}$ and Ghulam Rasool Mashori ${ }^{2}$ \\ ${ }^{1}$ Department of Applied pharmacologist, Italy \\ ${ }^{2}$ Department of Medical \& Health Sciences for Woman, Peoples University of Medical and Health Sciences for Women, Pakistan \\ ${ }^{*}$ Corresponding author: Luisetto M, Applied Pharmacologist, Hospital Pharmacist Manager, 29121 Italy \\ Submission: 海 October 06, 2017; Published: 漈 November 07, 2017
}

\begin{abstract}
Observing relevant biomedical literature we have see that clinical pharmacist play a crucial role in ICU settings with reducing in mortality rate and improving some clinical outcomes.
\end{abstract}

Keywords: ICU; Clinical pharmacy; Pharmaceutical care; Clinical outcomes; Mortality rate

\section{Introduction}

In ICU settings we can easily observe that the mortality rate is higher than other wards and for this reason a real multisiciplinatity medical team with added clinical ph. Competences can improve this situation. High intensity of cure, poly-pharmacy, critical patient conditions need also pharmaceutical competencies to be added to the classic decision making systems (clinical- managerial). The critically ill patients need a more rational decision making systems to improve clinical outcomes and in safety way.

\section{Material and Methods}

In this review and research paper we have searched some relevant biomedical literature in order to evaluate the efficacy of clinical pharmacist in improving clinical outcomes and reducing mortality rate. Then we observe the result of a practical experience (collaboration between emergency dep, hosp pharmacy, informatics engineered other healthcare professionals) involved in the management of an emergency ICU - HOSPITAL drug cabinet system.

\section{Results}

From literature published (period 1999-2017) we have found:

i. According Bond et al. [1] "A multivariate regression analysis, controlling for severity of illness, was employed to determine the associations. Four clinical pharmacy services were associated with lower mortality rates: clinical research $(\mathrm{p}<0.0001)$, his is the first study to indicate that both centrally based and patient-specific clinical pharmacy services are associated with reduced hospital mortality rates. This suggests that these services save a significant number of lives in our nation's hospitals."

ii. And in a JAMA article [2] we can see that "Pharmacist review of medication orders in the intensive care unit (ICU) has been shown to prevent errors, and pharmacist consultation has reduced drug costs. A senior pharmacist made rounds with the ICU team and remained in the ICU for consultation in the morning, and was available on call throughout the day.

The rate of preventable ordering ADEs decreased by $66 \%$ from 10.4 per 1000 patient-days (95\% confidence interval [CI], 7-14) before the intervention to $3.5(95 \% \mathrm{CI}, 1-5 ; \mathrm{P}<.001)$ after the intervention. In the control unit, the rate was essentially unchanged during the same time periods: 10.9 (95\% CI, 6-16) and $12.4(95 \% \mathrm{CI}$, 8-17) per 1000 patient-days.

The presence of a pharmacist on rounds as a full member of the patient care team in a medical ICU was associated with a substantially lower rate of ADEs caused by prescribing errors. Nearly all the changes were readily accepted by physicians".

iii. Gentry et al. [3] writed that: The clinical outcomes and cost-effectiveness of an antimicrobial control program (ACP) were studied. "Multivariate logistic regression models were constructed for mortality and for lengths of stay of 12 or more days. The acquisition costs of intravenous antimicrobial agents for the second baseline year and the entire program period were tabulated and compared. The average daily inpatient census was determined. The ACP was associated with a 2.4-day decrease in length of stay and a 
reduction in mortality from $8.28 \%$ (control) to $6.61 \%$ (intevenction) (p0,01) than intravenous antimicrobials decreased an average of only $5.7 \%$ over the two program years, but the acquisition cost. An ACP directed by a clinical pharmacist trained in infectious diseases was associated with improvements in inpatient length of stay and mortality. The ACP decreased intravenous antimicrobial costs and facilitated the approval process for restricted and non-formulary antimicrobial agents."

iv. Bond et al. [4] showed that "Education of medical and nursing staff, particularly by clinical pharmacists, is a vital part of a strategy to prevent medication errors". "That figure tended to have the greatest association (slope) with reductions in mortality rate, drug costs, and length of stay. As clinical pharmacist staffing levels increased from the tenth percentile $(0.34 / 100$ occupied beds) to the ninetieth percentile (3.23/100 occupied beds), hospital deaths declined from $113 / 1000$ to $64 / 1000$ admissions (43\% decline). This resulted in a reduction of 395 deaths/hospital/year when clinical pharmacist staffing went from the tenth to the ninetieth percentile. This translated into a reduction of 1.09 deaths/day/ hospital having clinical pharmacy staffing between these staffing levels, or 320 dollars of pharmacist salary cost/death averted. Three hospital pharmacy variables were associated with reduced length of stay in 1024 hospitals: drug protocol management (slope-1.30, $\mathrm{p}=0.008$ ), pharmacist participation on medical round (slope-1.71, $\mathrm{p}<0.001$ ), and number of clinical pharmacists/ occupied bed (slope-26.59, p<0.001). As drug costs/occupied bed/year increased, severity of illness-adjusted mortality rates decreased (slope-38609852, R(2) 8.2\%, p<0.0001). As the total cost of care/occupied bed/year increased, those same mortality rates decreased (slope -5846720642, R (2) 14.9\%, p<0.0001). Seventeen clinical pharmacy services were associated with improvements in the four variables."

v. Kane et al. [5] "Pharmacists are integral members of this team. They make valuable contributions to improve clinical, economic, and humanistic outcomes of patients. Pharmacist interventions include correcting/clarifying orders, providing drug information, suggesting alternative therapies, identifying drug interactions, and therapeutic drug monitoring. Pharmacist involvement in improving clinical outcomes of critically ill patients is associated with optimal fluid management and substantial reductions in the rates of adverse drug events, medication administration errors, and ventilator-associated pneumonia."

vi. According Rothschild [6]: We conducted a prospective 1-year observational study. "Most serious medical errors occurred during the ordering or execution of treatments, especially medications (61\%; 170/277). Performance level failures were most commonly slips and lapses (53\%; 148/277), rather than rule-based or knowledge-based mistakes. Adverse events and serious errors involving critically ill patients were common and often potentially life-threatening"

vii. Bond et al. [7]. In 2007clinical pharmacy service, pharmacy staffing, and hospital mortality rates. "In seven hospitals, clinical pharmacy service reduces mortality rates in a significant way". viii. MacLaren et al. [8] In this retrospective database review". In hospitals with ICU clinical pharmacy services, mortality rates in patients with TIE only and TIE with bleeding complications were higher by $37 \%$ (odds ratio [OR] 1.41, 95\% confidence interval [CI] 1.36-1.46) and 31\%(OR 1.35, 95\% CI 1.13-1.61), respectively, than in ICUs with clinical pharmacy services. Without clinical pharmacy services, bleeding complications increased by 49\%(OR 1.53, 95\% CI 1.46-1.60), resulting in 39\% more patients requiring transfusions (OR 1.47,95\% CI 1.28-1.69); these patients also received more blood products (mean \pm SD $6.8 \pm 10.4 \mathrm{v} / \mathrm{s} 3.1 \pm 2.6$ units/patient, $\mathrm{p}=0.006$ ) Involving clinical pharmacists in the direct care of intensive care patients with TIE was associated with reduced mortality, improved clinical and charge outcomes, and fewer bleeding complications. Hospitals should promote direct involvement of pharmacists in the care of patients in the ICU".

ix. MacLaren et al. [9] writed that: "Compared to ICUs with clinical pharmacists, mortality rates in ICUs that did not have clinical pharmacists were higher by $23.6 \%$ (p < 0.001, 386 extra deaths), $16.2 \%(\mathrm{p}=0.008,74$ extra deaths $)$, and $4.8 \%(\mathrm{p}=0.008$, 211 extra deaths) for nosocomial-acquired infections, communityacquired infections, and sepsis, respectively. The involvement of clinical pharmacists in the care of critically ill Medicare patients with infections is associated with improved clinical and economic outcomes. Hospitals should consider employing clinical ICU pharmacists."

x. Valentin et al. [10] observed that: "Observational, prospective, ( $0.9 \%$ of the studied) experienced permanent harm or died because of therapy errors at the administration. Parenteral medication errors at the administration stage are common and a serious safety problem in intensive care units. With the increase of the complexity of therapy in critically ill patients, the organization factors (as error reporting systems and routine checks) reduce the risk for this kind of errors."

xi. Chisholm et al. [11], 2010 in "Pharmacist's effect as team members on patient care: systematic review and meta-analyses": pharmacists provided direct patient care has favorable effects across various patient outcomes, health care settings, and disease states. (Significant p 0.005)."

xii. Shulman et al. [12] writed: "A prospective observational study Interventions were scored as low impact, moderate impact, high impact, and life saving. The final coding was moderated by blinded independent multidisciplinary trialists. This resulted in an overall intervention rate of $16.1 \%$ : $6.8 \%$ were classified as medication errors, $8.3 \%$ optimizations, and $1.0 \%$ consults. The interventions were classified as low impact (34.0\%), moderate impact (46.7\%), and high impact (19.3\%); and 1 case was life saving. Almost three quarters of interventions were to optimize the effectiveness of and improve safety of pharmacotherapy. This observational study demonstrated that both medication error resolution and pharmacist-led optimization rates were substantial. Two thirds of the interventions were of moderate to high impact".

xiii. Hisham [13], "In ICU poly-pharmacy is a very common. A total of 986 pharmaceutical interventions due to drug-related 
problems were documented, whereof medication errors accounted for $42.6 \%(n=420)$, drug of choice problem $15.4 \%(n=152)$, drugdrug interactions were $15.1 \%(\mathrm{n}=149), \mathrm{Y}$-site drug incompatibility was $13.7 \%(n=135)$, drug dosing problems were $4.8 \%(n=47)$, drug duplications reported were $4.6 \%(n=45)$, and adverse drug reactions documented were $3.8 \%(n=38)$. Drug dosing adjustment done by the clinical pharmacist included $140(11.9 \%)$ renal dose, 62(5.2\%) hepatic dose, 17(1.4\%) pediatric doses, and $104(8.8 \%)$ insulin dosing modifications. Clinical pharmacist as a part of multidisciplinary team in our study was associated with a substantially lower rate of adverse drug event caused by medication errors, drug interactions, and drug incompatibilities."

xiv. Luisetto et al. [14] writed that (2017) "We think that the clinical ph. main focus must be involved in priority way to the most critical patients in order to achieve the best results available. In this condition even benefit of 1 life achieved in mortality rate is a real golden endpoint (we can think for example to a pediatric poisoning, or severe infectious disease in pregnancy or the effect of inefficacy immunosuppressive therapy in transplanted at other). This can be considered in example as a reduction in NNT to improve a therapeutic strategy."

xv. According Amine Ali Zeggwagh et al. [15] "The present study demonstrated that the preventable in-ICU deaths are a serious problem occurring in $14.1 \%$ of all deaths observed in our ICU."

xvi. Dilip Kothari et al. [16] writed that "Medication error is a major cause of morbidity and mortality in medical profession, and anesthesia and critical care are no exception to it. Man, medicine, machine and modus operandi are the main contributory factors to it. In this review, incidence, types, risk factors and preventive measures of the medication errors are discussed in detail".

xvii. Eric Moyen et al. [17] "Pharmacists, similarly, have an crucial role to play in medication safety. First, all intravenous medications should be prepared within the pharmacy department by pharmacists using a standardized process and standardized medication concentrations. Second, participation of a pharmacist in clinical rounds improves patient safety by reducing preventable ADEs by $66 \%$ (61) while shortening patients' length of hospital stay $(62,63)$, decreasing mortality $(64)$, and decreasing medication expenditures."

Amine Ali Zeggwagh, et al. [15] "In general, following things should be kept in mind while working in the operation room to minimize the incidence of medication errors:

a) Reducing the complexity of the system to simple and linear to enhance the safety

b) Redundancy and standardization are the basic principles in the design of a safe system

c) Double checking of ampoules, syringes and equipment before starting the procedure

d) Simple vigilance during the handling and administration of drugs is of utmost importance
After a systemic review, Jenson and colleagues (55) recommended a 12-point strategy to prevent medication errors during anesthesia and critical care:

The label on any drug ampoule or syringe should be read carefully before the drug is drawn up or injected. Legibility and contents of labels on ampoules and syringes should be optimized according to agreed standards with respect to font, size, colour and information. Syringes should always be labeled. Formal organization of drug drawers and work space should be used with attention to tidiness, position of ampoules and syringes, separation of look-alike drugs and removal of dangerous drugs from the operation room. Labels should be checked specifically with the help of a second person or a device like bar code reader before administration. Error during administration should be reported and reviewed. Management of inventory should focus on minimizing the risk of drug error. Lookalike packaging and presentation of the drug should be avoided where possible. Drug should be presented in prefilled syringes rather than ampoules. Drug should be drawn up and labeled by the anesthesia provider himself/herself. Colour coding by class of drugs should be according to an agreed national or international standard. Coding of syringe according to position or size should be done.

Several other measures to promote safe drug administration during anesthesia and critical care have been suggested. The provision of all labels in a standardized format emphasizing the class and generic name of each drug incorporating the bar code and class-specific colour code as per international standard. The bar code reader use to detect the drug at the point of administration immediately way before it is given linked to an auditory prompt to facilitate the checking control of the drug identity. Integration of scanned information into an automated anesthesia record and reducing the cognitive load on the anesthetist. The use of medical devices at the point of care to automatically measure the dose of the administered pharmacological drug. A dosing monograph on the infusion syringe label to avoid the need of look-up tables or dose calculations. The automatized drug dispensing system with features such as single issue drawers and bar code scanners to facilitate safer dispensing of the pharmacological drugs.

Camire et al. in a review article have suggested seven strategies to prevent errors in ICU. These are as follows: Eliminating extended physician work schedules. Computerized physician order entry. Implement support system for clinical decisions. Computerized intravenous devices. Active participation of pharmacists in ICU. Medication reconciliation.

Merali et al. made many recommendations to reduce medication errors at different stages. Recommendations to reduce medication errors. Many organizations are now dedicated to patient safety."

xviii. According Fideles et al. [18] "The number of PhRs considered exerting high impact on pharmacological strategy increased in the last period of the study, including those concerning dose adjustment, treatment discontinuation, and recommendation of treatment onset. This finding might be due to improvement of the 
clinical knowledge of the pharmacist and his/her more thorough integration with the ICU multi-professional staff. According to the BIOMEDICAL literature, pharmacist's actions in the ICU should not to be limited to providing advice to equip but also include pro- active participation in decision-making in the maintenance of pharmacological treatment."

xix. Preslaski et al. [19] showed that "Augmented by ICT technology and resource Use, to provide valuable services in the form of assisting physicians and clinicians with pharmacotherapy decision-making, reducing drug therapy errors, and improving medication safety systems to optimize patient outcomes. The addition of a pharmacist to inter professional critical care team should be encouraged as health-care systems focus on improving the quality and efficiency of care delivered to improve patient outcomes."

xx. Calloway et al. [20] "Health care organizations are turning to electronic clinical decision support systems (CDSSs) to increase quality of patient care and promote a safer environment. Prior to CDSS implementation, clinicians struggled with obtaining and documenting the data needed to support clinical initiatives. The value of having both clinical and staff pharmacists utilizing the CDSS has improved communication and knowledge among staff and improved relationships with medical staff, nursing, and case management. The department of pharmacy increased its clinical interventions from an average of 1,986 per month to 4,065 per month; this represents a $105 \%$ increase in the number of interventions".

xxi. Kucukarslan et al. [21] "To determine the impact of a pharmacist who is permanently assigned to the medical intensive care unit (MICU) on the incidence of preventable ADEs, drug charges, and length of stay (LOS) in the MICU.

A randomized, experimental versus historical control group design was used. Preventable ADEs were identified and validated by 2 pharmacists and a critical care physician. Information about MICU drug charges and LOS were obtained from the hospital administrative database. The intervention group had fewer occurrences of ADEs (10 ADEs/1,000 patient days) when compared to the control group (28 ADEs/1,000 patient days) at a significance level of .03. No significant differences were found between the 2 groups in MICU drug charges and LOS. The vast majority of the 596 documented recommended interventions (99\%) were accepted by the medical team. Nutrition monitoring, medication indicated but not prescribed, and dosage modification were the top 3 problems identified by the pharmacist.

The addition of a dedicated critical care pharmacist to the MICU medical team improves the safe use of medication.

xxii. Rudis et al. [22] writed: "By combining the strengths and expertise of critical care pharmacy specialists with existing supporting literature, these recommendations define the level of clinical practice and specialized skills that characterize the critical care pharmacist as clinician, educator, researcher, and manager. This Position Paper recommends fundamental, desirable, and optimal pharmacy services as well as personnel requirements for the provision of pharmaceutical care to critically ill patients".

xxiii. Randolph et al. [23] "Critically ill patients are at high risk for death and permanent disability. Their care is also very expensive. The method of delivering critical care services to these patients can have an impact on their clinical and economic outcomes. Researchers face significant challenges when attempting to identify an association between an intensive care unit (ICU) organizational characteristic and patient outcomes. In this paper we review the challenges faced when evaluating the impact of ICU organizational characteristics on patient outcomes and highlight ICU characteristics that are consistently associated with improved patient outcomes.

These characteristics include:

(i) The presence of specialist physicians devoted to the ICU;

(ii) Increased nurse: patient ratios;

(iii) Decreased use of tests and evaluations that will not change clinical management;

(iv) Development and implementation of evidence-based protocols and guidelines;

(v) Use of computer-based alerting and reminding systems; and

(vi) Having a pharmacist participate in daily rounds in the ICU.

When implementing these in ICUs, it is important to evaluate the impact of these characteristics on patient outcomes. We provide a format for such an evaluation. Given the growing evidence supporting the association between specific ICU characteristics and improved patient outcomes, we hope the future realizes broad implementation of these beneficial characteristics".

xxiv. Kruer et al. [24] "The Institute of Medicine has reported that medication errors are the single most common type of error in health care, representing $19 \%$ of all adverse events, while accounting for over 7,000 deaths annually. The frequency of medication errors in adult intensive care units can be as high as 947 per 1,000 patient-days, with a median of 105.9 per 1,000 patient-days. The formulation of drugs is a potential contributor to medication errors. Challenges related to drug formulation are specific to the various routes of medication administration, though errors associated with medication appearance and labeling occur among all drug formulations and routes of administration. Addressing these multifaceted challenges requires a multimodal approach. Changes in technology, training, systems, and safety culture are all strategies to potentially reduce medication errors related to drug formulation in the intensive care unit."

\section{A Practical experience:}

Emergency drug hospital cabinet systems practical experiencePC (society of Italian hospital pharmacist POSTER ABSTRATC) MILAN-national congress2016 (this poster has win the best presentation award in young pharmacist section). [25] 
In this example the ICT tool can make possible to have the real situation of the emergency drug (quali- quantitative) in every SPOKE and rapid information about emergency drugs position in order to achieve they in rapid way to have availability of other emergency drugs(h24 also with central pharmacy closed) in a safety way and containing costs. This experience is linked with a correct management of the systems with a really rational way and recognized officially by SIFO society of hospital pharmacist (Italy) in this practical experience [13] no near miss event or other patient risk or even fatal event was observed related to emergency drugs stokes.

During 6 month it was covered the emergency need of drugs in the $99 \%$ of cases, and only 1 time was necessary the central pharmacy call by night or in week end. (The same results was observed during 1 year) (Provincial public hospital with about 700 beds, 4 hospitals linked). This kind of project was introduced by a multidisciplinary team according a risk management and ICT Approach. (Emergency and ICU clinicians, clinical pharmacist, informatics, engineers, nurse, toxicological med lab professionals and other). This system in fact make possible to know rapidly the situation of expiration time of all emergency drugs stoked and so make more easy the ordering process to have continuity in providing.

\section{Discussion}

From the analysis of the cited literature we can say that: The pharmacy services are associated to reduce mortality rate with a low rate in ADES by prescribing errors. That in an antimicrobial control program with clinical pharmacist involved it was obtained a reduction in mortality rate in significative way $(p=001)$ [3]. That the mortality rate involved in clinical pharmacist in medical team reduced hospital deaths about 43\% [4]. Medications are heavily involved in most serious medical errors in many hospital clinical ph. Service reduced mortality rate in significant way [6] clinical pharmacist involved in patient therapy with infectious disease improved clinical outcomes related to the therapy of critically ill patients in ICT organizational factor can reduce risk for errors [23] that related a systematic review and met-analysis various favorable effect on patient's outcome by pharmacists effective member of medical team [11] in reviewing of therapies the pharmacist intervenctions was classified as HIGH IMPACT (19.3\%) [12] and that poly-pharmacy is very common in ICU SETTINGS is very common (obviously).

And at least according the editorial the clinical pharmacist main focus " the clinical pharmacist must be applied in priority way to the most critical patients to achieve the best results available " and " this can be considered as a reduction in NNT to improve a therapeutic strategy.[14]

Related to the results of a a practical experience (emergency drug cabinet systems) we have see during 1 year no near miss event or other patient risk using an ICT system managed by clinical pharmacist to cover hospital need of emergency drugs placed in ICU WARD [24]. (An example of collaboration with emergency dep, ICU, hospital pharmacy and blood bank and biomedical engineer software house. This kind of results obtained by literature confirms the fundamental role (and not ancillar) of the clinical pharmacist in ICU Medical Team.

\section{Conclusion}

Observing the results of the literature reported in this paper we can say that the clinical pharmacist presence in stabile way in ICU medical team gives improving in some clinical outcomes and reducing mortality rate. This conclusion is related to the complexity of ICU SETTINGS and by critical patient's condition. To adeguately managed this situations are needed the most complete medical equips (multi-disciplinariety). We observe that the role played by hospital pharmacists can be in more clinical activities, as educator (towards all healthcare professionals), researcher, and manager functions.

\section{References}

1. Bond CA, Raehl CL, Franke T (1999) Clinical pharmacy services and hospital mortality rates. Pharmacotherapy 19(5): 556-564.

2. Leape LL , Cullen DJ, Clapp MD, Burdick E, Demonaco HJ (1999) Pharmacist participation on physician rounds and adverse drug events in the intensive care unit. JAMA 282(3): 267-270.

3. Gentry CA, Greenfield RA, Slater LN, Wack M, Huycke MM (2000) Outcomes of an antimicrobial control program in a teaching hospital. Am J Health Syst Pharm 57(3): 268-274.

4. Bond CA, Raehl CL, Franke T (2001) Interrelationships among mortality rates, drug costs, total cost of care, and length of stay in United States hospitals: summary and recommendations for clinical pharmacy services and staffing. Pharmacotherapy 21(2): 129-141.

5. Kane SL, Weber RJ, Dasta JF (2003) The impact of critical care pharmacists on enhancing patient outcomes. Intensive Care Med 29(5): 691-698.

6. Rothschild JM, Landrigan CP, Cronin JW, Kaushal R, Lockley SW, et al. (2005)The Critical Care Safety Study: The incidence and nature of adverse events and serious medical errors in intensive care. Crit Care Med 33(8):1694-1700.

7. Bond CA et al. (2007) Clinical pharmacy service, pharmacy staffing, and hospital mortality rates. "In seven hospitals, clinical pharmacy service reduces mortality rates in a significant way.

8. MacLaren R, Bond CA (2009) Effects of pharmacist participation in intensive care units on clinical and economic outcomes of critically ill patients with thromboembolic or infarction-related events. Pharmacotherapy 29(7): 761-768.

9. MacLaren R, Bond CA, Martin SJ, Fike D (2008) Clinical and economic outcomes of involving pharmacists in the direct care of critically ill patients with infections. Crit Care Med 36(12): 3184-3189.

10. Valentin A, Capuzzo M, Guidet B, Moreno R, Metnitz B, et al. (2009) Errors in administration of parenteral drugs in intensive care units: multinational prospective study. BMJ 338: b814.

11. Chisholm-Burns MA, Kim Lee J, Spivey CA, Slack M, Herrier RN, et al. (2010) US pharmacists' effect as team members on patient care: systematic review and meta-analyses. Med Care 48(10): 923-933.

12. Shulman R, McKenzie CA, Landa J, Bourne RS, Jones A, et al. (2015) Pharmacist's review and outcomes: Treatment-enhancing contributions tallied, evaluated, and documented (PROTECTED-UK). J Crit Care 30(4): 808-813.

13. Hisham M, Sivakumar MN, Veerasekar G (2016) Impact of clinical pharmacist in an Indian Intensive Care Unit. Indian J Crit Care Med 20(2): 78-83. 
14. Luisetto M, Nili-Ahmadabadi B, Mashori GR (2017) The Clinical Pharmacists Main Focus. J Appl Pharm 9(4): e114.

15. Amine Ali Zeggwagh, HoudaMouad, TarekDendane, Khalid Abidi, JihaneBelayachi, et al. (2014) Preventability of death in a medical intensive care unit at a university hospital in a developing country. Indian J Crit Care Med 18(2): 88-94.

16. Dilip Kothari, Suman Gupta, Chetan Sharma, Saroj Kothari (2010) Medication error in anaesthesia and critical care: A cause for concern. Indian J Anaesth 54(3): 187-192.

17. Eric Moyen, Eric Camiré, Stelfox HT (2008) Clinical review: Medication errors in critical care. Crit Care 12(2): 208.

18. Fideles GM, de Alcântara-Neto JM, Peixoto Júnior AA, de Souza-Neto PJ, Tonete TL, et al. (2015) Pharmacist recommendations in an intensive care unit: three-year clinical activities. RevBras Ter Intensiva 27(2): 149-154.

19. Preslaski CR, Lat I, MacLaren R, Poston J (2013) Pharmacist contributions as members of the multidisciplinary ICU team. Chest 144(5): 1687-1695.
20. Calloway S, Akilo HA, Bierman K (2013) Impact of a clinical decision support system on pharmacy clinical interventions, documentation efforts, and costs. Hosp Pharm 48(9): 744-752.

21. Kucukarslan SN, Corpus K, Mehta N, Mlynarek M, Peters M, et al. (2013) Evaluation of a dedicated pharmacist staffing model in the medical intensive care unit. Hosp Pharm 48(11):922-930.

22. Rudis MI, Brandl KM (2000) Position paper on critical care pharmacy services. Society of Critical Care Medicine and American College of Clinical Pharmacy Task Force on Critical Care Pharmacy Services. Crit Care Med 28(11): 3746-3750.

23. Randolph AG, Pronovost P (2002) Reorganizing the delivery of intensive care could improve efficiency and save lives. J Eval Clin Pract 8(1): 1-8.

24. Kruer RM, Jarrell AS, Latif A (2014) Reducing medication errors in critical care: a multimodal approach. ClinPharmacol 6: 117-126.

25. (2016) Emergency drug hospital cabinet systems practical experiencePiacenza (SIFO society of Italian hospital pharmacist Poster abstract) 2016 MILAN GIORNALE ITALIANO DI FARMACIA CLINICA, Acts of the XXXVII Congress National SIFO Milan. 